\title{
Effect of mutagens on regeneration and growth of in vitro grown epicotyl segments of rough lemon seedlings (Citrus jambhiri Lush.)
}

\author{
Sukhjit Kaur \\ Horticulture Section, Punjab Agricultural University, Regional Research Station \\ Gurdaspur-143521, (Punjab), INDIA \\ E-mail: sukhi.rose@gmail.com \\ Received: January 14, 2014; Revised received: May 21, 2015; Accepted: June 5, 2015
}

\begin{abstract}
The present study on the effect of mutagens on regeneration and growth of in vitro grown epicotyls segments of rough lemon seedlings (Citrus jambhiri Lush.) was carried out during the years 2009- 2010 and 2010- 2011 in the Tissue Culture Laboratory, Department of Fruit Science, Punjab Agricultural University, Ludhiana. Developmental characteristics of the in vitro grown epicotyls segments on regeneration media were treated employing gamma radiation in Gray(Gy) at 0, 5,10,15,20,25,30,35,40 and 45 Gy; the alkylating agent ethyl methane sulfonate (EMS) and methyl methane sulfonate (MMS) each at $0,0.1,0.2,0.3,0.4,0.5$ and $0.6 \%(\mathrm{v} / \mathrm{v})$ were evaluated. Epicotyl segments from one month old in vitro grown seedling were cultured in regeneration medium (MS+BAP 1.0 $\left.\mathrm{mglit}^{-1}\right)$ under controlled laboratory conditions $\left(25 \pm 2^{0} \mathrm{C}, 16 \mathrm{hr}\right.$ photoperiod, 2000 lux light). $\mathrm{LD}_{50}$, the dose required to kill half of the tested population corresponded to 35Gy for gamma radiation, $0.3 \%$ each for EMS and MMS treatments. Number of days taken for regeneration increased with increasing dose of gamma irradiation, EMS and MMS. Percent regeneration, number of buds, number of shoots, shoot length, number of leaves, internodal length, primary root length and number of secondary roots decreased with increasing dose of gamma radiation, EMS and MMS. The study would be beneficial to induce desirable variations in plant growth characteristics of rough lemon by the use of mutagens treatment.
\end{abstract}

Keywords: Ethyl methane sulfonate, Gamma rays, Germination, In vitro, Methyl methane sulfonate, Rough lemon

\section{INTRODUCTION}

In vitro mutagenesis is considered as a valid tool for the improvement of a crop, especially when we have to add one or more easily identifiable characters without changing the genotype of well developed variety. In addition, there is no loss of the mutants, as micropropagules are sub-cultured under sterile conditions (Ling et al., 2008). At the current level of plant breeding research, the mutation breeding is highly suitable as compared to natural variation. Mutation breeding is more effective than hybridization even when desired genes are present, but tightly to undesirable genes. The frequency of occurrence of mutation by the use of mutagen may as higher as 300 times than the occurrence of natural frequency seen (Wan et al., 1991).Hence, attempts have been made to accelerate the rate artificially using physical and chemical mutagens. The frequency and saturation of mutations can be regulated by varying the mutagen dose (Jander et al., 2003; Kim et al., 2006) and mutagenic agents can induce different extensions of genomic lesions, ranging from base mutation to larger fragments insertions or deletions(Mackenzie et al., 2005; Kim et al., 2006).

In plants, the response to physical and chemical mutagens is species-specific and largely unknown for the majority of the species (Gilchrist and Haughn, 2005). Several workers have attempted for induction of mutation in citrus species using either physical and chemical mutagens for involving new citrus genotypes like seedlessness in sweet orange and grape fruit cultivars (Davis and Albligo, 1994) and salt tolerance in troyer citrange (Garcia -Agustin and Primo-Millo, 1995), development of seedless and Mal Secco tolerant mutant lemons (Gulsen et al., 2007), seedless and citrus canker tolerant mutant clones in sweet orange (Latado et al., 2006). In mutagenesis , mutagens viz., physical(gamma rays) and chemicals such as ethyl methane sulfonate (EMS) and methyl methane sulfonate (MMS) are most frequently used (Jain, 2005). Citrus is a plant that have long juvenility period and breeding of which is restricted by conventional methods due to complication of their genetic systems (Kayim and Koe, 2006). Although several citrus rootstocks have been propagated through tissue culture, very limited work are reported on in vitro multiplication of rough lemon (Chaturvedi et al., 2001). Hence the availability of an efficient regeneration system is also a pre-requisite for genetic improvement and genetic resource conservation. In the present study, 
gamma rays in Gray (Gy), ethyl methane sulfonate (EMS) and methyl methane sulfonate (MMS) have been used with the objective to study the effect of various mutagenic treatments on in vitro regeneration and different growth parameters of rough lemon seedlings.

\section{MATERIALS AND METHODS}

Seeds of rough lemon were obtained from citrus germplasm block of college orchard of Punjab Agricultural University, Ludhiana during the years 2009-10 and 2010-11. Nine increasing dose of gamma irradiations $(0,5,10,15,20,25,30,35,40$ and 45Gy), EMS $(0,0.1,0.2,0.3,0.4,0.5$ and $0.6 \%)$ and MMS $(0,0.1,0.2,0.3,0.4,0.5$ and $0.6 \%)$ were evaluated for epicotyls segments of rough lemon fully randomized in five replicates of 24 epicotyl segments $(n=120)$.

After removing the seed coat, seeds were surface sterilized with mercuric chloride $(0.1 \%)$ solution for 4 minutes and then the seeds were rinsed thrice with autoclaved distilled water to remove the traces of mercuric chloride under Laminar Air Flow Cabinet. After sterilization, seeds were sown in Murashige and Skoog (1962) basal medium in culture jars. These cultured jars were incubated at $25 \pm 2^{\circ} \mathrm{C}$ temperature in dark for two weeks for etiolation. Germination of seeds starts after 6-7 days after sowing. After 4-5 weeks these culture jars were shifted to light for 16 hours continuous fluorescent white light (2000lux) followed by a dark period of 8 hours. Epicotyl segments(1-2cm long) in vitro grown one month old seedlings of rough lemon were cultured on direct regeneration (MS+BA @ $1 \mathrm{mglitre}^{-1}$ ) media in petri plates and submitted to gamma rays from the Cobalt ${ }^{60}$ source and then shifted to the culture jars containing same regeneration media under controlled laboratory conditions $\left(25 \pm 2^{\circ} \mathrm{C}, 16\right.$ hours photoperiod and 2000 lux light). Chemical mutagenesis was carried out by immersing the epicotyls segments from in vitro grown one month old seedlings in filter sterilized $\operatorname{EMS}(0,0.1,0.2,0.3,0.4,0.5$ and $0.6 \%)$ and $\operatorname{MMS}(0,0.1,0.2,0.3,0.4,0.5$ and $0.6 \%)$ for 4 hours in an incubator shaker $\left(25 \pm 2^{\circ} \mathrm{C}, 70\right.$ r.p.m. $)$. Then epicotyls segments were rinsed with autoclaved distilled water thrice to remove the traces of mutagens. Treated epicotyls segments along with control (120 epicotyl segments per treatment) in each set of experiments (EMS and MMS) were cultured in culture jars containing direct regeneration media (MS+ BA@1mglitre $\left.{ }^{-1}\right)$ under controlled laboratory conditions $\left(25 \pm 2^{\circ} \mathrm{C}, 16\right.$ hour photoperiod and 2000lux light). In above three experiments, data was recorded for number of days taken for regeneration, per cent regeneration ,number of buds ,number of shoots, shoot length $(\mathrm{cm})$,number of leaves, internodal length $(\mathrm{cm})$, length of main roots $(\mathrm{cm})$ and number of secondary roots. $\mathrm{LD}_{50}$ doses were optimized by taking into account the regeneration of epicotyl segments. The forecast analysis (Microsoft Exel) was used to calculate the lethal mutagen dosage required to kill half of the popu- lation $\left(\mathrm{LD}_{50}\right)$. Percent regeneration was calculated by standard procedure 6-7 days after culturing. Shoot length $(\mathrm{cm})$ and internodal length $(\mathrm{cm})$ were measured with Vernier's Calliper, 120 days after culturing. The experiment was laid in Completely Randomized Block Design (CRD) as described by Singh et al. (1998). Microsoft Exel version 2007 was used for statistical analysis.

\section{RESULTS AND DISSCUSION}

Effect of gamma radiation: Citrus plant has several natural factors as cause of variability (Ribeiro and Machado, 2007). In vitro regeneration of epicotyls segments was (significantly at 5\% level) decreased with increasing dose of gamma radiations (Table 1). The earliest regeneration was observed in 0Gy (control) (7.21 days) followed by 5Gy (10.41 days), 10Gy (12.0 days), 15Gy (12.50days), 20Gy (15.52days), 25Gy (16.00days), 30Gy (18.61days), 35Gy (20.51days), 40Gy (22.20days) and 45Gy (25.21days) treatment. Mean regeneration percentage was highest in control (98.0) followed by 5Gy (90.51), 10Gy (80.25), 15Gy (75.41), 20Gy (70.30), 25Gy (66.52), 30Gy (56.50), 35Gy (48.50), 40Gy (44.12) and $45 \mathrm{~Gy}(30.00)$ treatments.

The reduction in germination percentage and delay in germination of Kinnow seeds due to gamma ray treatment was also reported by Dhatt et al. (2000) and Latado et al. (2001). Most of the ill effects of gamma radiation treatment followed immediately after treatment and were manifested in terms of decreased sprouting capacity with increase in the dose (Raghami and Ghazvini, 2005). Kumar and Mishra (2004) noted that in okra (Abelmoschus esculentus), germination percentage generally decreased with the increasing doses of gamma rays. Reduction in germination percentage with increasing dose of gamma radiation has also been reported in Pinus (Thapa, 2004), Rye (Akgun and Tosum, 2004), Chickpea (Khan et al., 2005), Cicer (Toker et al., 2005) and Citrus jambhiri Lush. (Sharma et al., 2013; Saini and Gill, 2009; Kaur and Rattanpal, 2010).

Mean shoot length and internodal length (significantly at $5 \%$ level) decreased with increasing dose of gamma radiations(Table 1).The maximum shoot length and internodal length were observed in control $(5.83 \mathrm{~cm}$ and $2.20 \mathrm{~cm}$ respectively) and minimum in $45 \mathrm{~Gy}$ $(2.30 \mathrm{~cm}$ and $1.20 \mathrm{~cm}$ respectively). Similarly, Kerkadze (1985) and Khokhar (1998) observed the decrease in mean seedling height and internodal length with increasing gamma radiation doses in citrus. Reduction in plant growth and shoot length was also reported in kinnow seedlings (Legave et al.,1989; Waqar et al.,1992) and Citrus jambhiri Lush. seedlings (Kaur and Rattanpal, 2010). Radiation treatments probably induced certain changes at genetic level that ultimately get reflected in the substances that trigger biochemical processes controlling different aspects of 
the growth. Such substances were identified as auxins, gibberellins, ethylene and abscisic acid, called phytohormones, initiate biochemical reactions and induce changes in chemical composition, there occur changes in chemical patterns which lead to various modifications and variations in plant characters such as height, branching, stem thickness and flowering etcetera (Whittwer, 1971)

Similarly, number of buds, number of shoots, number leaves, root length and number of secondary roots (significantly at 5\% level) decreased with increase in dose of gamma radiations (Table 1). Maximum number of buds, shoots, leaves, secondary roots and root length $(35.10,30.15,10.50,17.12$ and $8.75 \mathrm{~cm}$ respectively) was recorded in control followed by $5 \mathrm{~Gy}$ and minimum $(12.00,8.51,3.50,7.00$ and $4.40 \mathrm{~cm}$ respectively) in 45 Gy treatment. Number of leaves and primary root length were also decreased with increasing dose of gamma radiation in Citrus jambhiri Lush. (Kaur and Rattanpal; 2010 Sharma et al., 2013).Reduction in number of leaves and branches in Kinnow (Khokhar,1998), and root growth and shoot elongation in grapefruit (Kawamura et al., 1989) was also observed with increasing dose of gamma rays. The radiation was reported to cause malfunctioning of various phyto-hormones and cause changes in chemical patterns leading to morphological variations by Swaminathan (1965). Radiation treatments also cause quantitative as well as qualitative alteration in the hereditary material. The morphological effects due to radiation have been reported in stem, leaves, branches and even fruits (Sparrow and Gunckel, 1956). Kaur and Rattanpal (2010) and Sharma et al. (2013) reported that in Citrus jambhiri Lush., with increasing dose of gamma radiations, the plant height, number of leaves and primary root length were decreased. Saini and Gill (2009) observed that in Citrus jambhiri Lush., with increasing dose of gamma radiation treatment, seedling height was decreased. These are generally recessive to the normal type, thereby suggesting that the mutations induced are due to destruction of the gene(s). The variability for number of leaves and number of branches per seedling was also reported in Chrysanthemum (Datta et al., 2005) and Lepium sativum ( Majeed et al., 2010). On the contrary, no such variability was reported by Jawaharlal et al. (1992) in acid lime thereby indicating varietals or genetic specificity of each genotype to radiations.

Effect of Ethyl methane sulfonate (EMS): The number of days taken for regeneration and percent regeneration were significantly affected at $5 \%$ level with increasing doses of EMS. (Table2). In control (0\%), epicotyls segments were regenerated in 7.00 days and regeneration percentage was 98.11. However with $0.1 \%$ EMS concentration, number of days taken for regeneration increased to 13.53 and germination reduced to 75.33. But seed germination was totally inhibited at $0.6 \%$ EMS dose. Similarly, the delay in seed germination as well as reduction in seed germination percentage with increasing EMS doses was also reported in acid lime (Jawaharlal et al.,1992), kinnow (Khokhar,1998) and Citrus jambhiri Lush (Kaur and Rattanpal,2010; Sharma et al., 2013) . The sprouting capacity of the seeds fall and they show poor germination after EMS treatment was reported due to the affect on cytochrome oxidase content, thus reducing the respiration and hence causing death of the seeds or delayed germination in barley and wheat (Swaminathan et al., 1962). The presoaking of seeds was also reported to increase the vulnerability of seeds to EMS. The actively dividing phase gets drastically affected so that no germination occur due to alteration of gene(s) or gene complexes (Singh and Singh, 1989). Chromosomal aberrations may also occur due to EMS treatment which prevents healthy and quicker seed germination.

Mean shoot length and internodal length decreased significantly at 5\% level with increasing dose of EMS. The maximum shoot length and internodal length were observed in control $(5.66 \mathrm{~cm}$ and $2.65 \mathrm{~cm}$ respectively) followed by $0.1 \%$ and minimum in $0.5 \%(2.51 \mathrm{~cm}$ and $0.71 \mathrm{~cm}$ respectively). The reduction in mean seedling height because of increasing treatment doses was also reported in apple (Sharma and Sharma, 1996), rice (Gupta and Sharma, 1994) and Citrus jambhiri Lush (Kaur and Rattanpal, 2010) . Khokhar (1998) recorded lower seedling height, internodal length, leaf number and number of branches per seedling in Kinnow mandarin. Mallick et al. (1978) suggested that variation in one or more characters might have been due to various mutagenic effects such as mutation of genes, breaking of tightly linked regions and crossing over within these regions, enhanced recombination, individual or a combination of two or more such effects. The depressing effect of EMS on seedling height and other characters in present study might have been due to other physiological damage or due to any of the reasons cited above.

Similarly number of buds, number of shoots ,number leaves, root length and number of secondary roots decreased significantly at 5\% level with increase in dose of EMS(Table2 ).Maximum number of buds, shoots, leaves, secondary roots and root length $(35.20,30.10,9.0,15.0$ and $8.50 \mathrm{~cm}$ respectively) was recorded in control followed by $0.1 \%$ and minimum $(10.00,4.20,3.31,3.15$ and $3.10 \mathrm{~cm}$ respectively) in $0.5 \%$ treatment. Similarly, Khokhar (1998) reported that with increase in the dose of EMS, number of leaves and branches were decreased. Variations for different morphological characters were probably due to phenotypically constructive multidirectional mutations of polygenes caused by mutagen EMS. The varying response of plants after EMS treatments may be attributed to the differential sensitivity of different loci among the genotype for same or different characters. With increasing dose of EMS treatment, the percent 

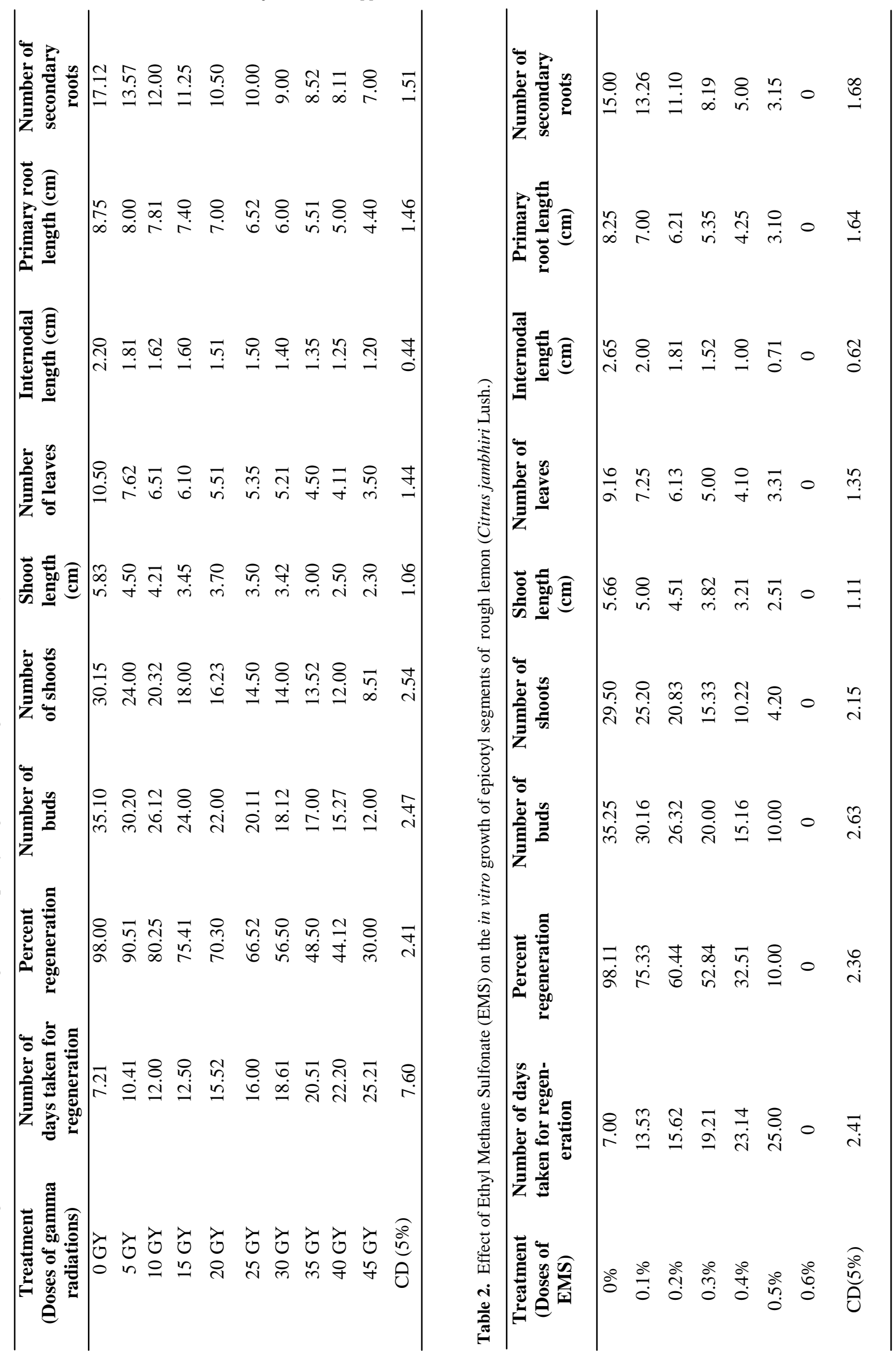
germination, plant height, internodal length, number of leaves and primary root length were decreased as reported in Citrus jambhiri Lush.( Kaur and Rattanpal, 2010 ; Sharma et al.,2013) .

Effect of Methyl methane sulfonate (MMS): The number of days taken for regeneration and percent regeneration were (significantly at 5\% level) affected with increasing doses of MMS (Table 3). Epicotyls segments were regenerated in 7.22 days and regeneration percentage was 97.00 in control $(0 \%)$. With $0.1 \%$ MMS concentration, number of days taken for regeneration increased to 14.00 and germination was $76.15 \%$. At $0.6 \%$ MMS dose, seed germination was totally inhibited.

Mean shoot length and internodal length decreased significantly at 5\% level with increasing dose of MMS. The maximum shoot length and internodal length were observed in control $(5.41 \mathrm{~cm}$ and $2.71 \mathrm{~cm}$ respectively) followed by $0.1 \%$ and minimum in $0.5 \%(2.40 \mathrm{~cm}$ and $0.60 \mathrm{~cm}$ respectively). Veleminsky et al. (1975) reported that MMS treatment to the non-germinating grains of barley also induced the reduction of germination and seedling height.

Similarly, number of buds, number of shoots ,number leaves, root length and number of secondary roots decreased significantly at $5 \%$ level with increase in dose of MMS (Table 3).Maximum number of buds, shoots, leaves, secondary roots and root length $(32.45,31.10,9.0,18.0$ and $8.62 \mathrm{~cm}$ respectively)was recorded in control followed by $0.1 \%$ and minimum $(8.0,3.22,3.0,3.0$ and 3.0 respectively) in $0.5 \%$ treatment. Gad and El-Sawah (1985), while working on peas observed that MMS treatment on dry seeds of peas reduced the seedling height with increasing dose. Sharma et al. (2013) reported that in Citrus jambhiri Lush., with increasing dose of MMS treatment, the percent germination, plant height, intermodal length, number of leaves and primary root length were decreased.

$\mathrm{LD}_{50}$, the dose required to kill half of the tested population corresponded to $35 \mathrm{~Gy}$ for gamma radiation, $0.3 \%$ each for EMS and MMS treatments as per forecast analysis done in Microsoft Excel(Table 1, 2 and 3 ). The $\mathrm{LD}_{50}$ value of gamma rays and EMS for Kinnow seeds was found to be around $10 \mathrm{kR}$ and 0.4 per cent, respectively by Dhatt et al. (2000). Waqar et al. (1992) reported it to be $10 \mathrm{kR}$ for Kinnow and Hearn (1984) found it to be between 10 and $15 \mathrm{kR}$ for Pineapple sweet orange seeds and $15 \mathrm{kR}$ for Duncan grapefruit seeds and thus concluded that $\mathrm{LD}_{50}$ is specific for each variety. Varying value of $\mathrm{LD}_{50}$ dose in different citrus cultivars was also reported by Hensz (1971). Similarly, Dhatt et al. (2000) reported that $\mathrm{LD}_{50}$ dose for Kinnow seed with gamma rays slightly less than 10 $\mathrm{kR}$.

\section{Conclusion}

The mutation treatments did not improve the epicotyl 
segments regeneration and seedlings growth in Citrus jambhiri Lush. It caused severe reduction in percent regeneration and seedling growth of epicotyls segments with increase in mutagens dose. Therefore, 35 Gy for gamma radiations and $0.3 \%$ each for EMS and MMS treatments might be considered as optimum dose $\left(\mathrm{LD}_{50}\right)$ for percent regeneration because beyond this dose, there was a gradual decrease in percent regeneration and seedling growth parameters.

\section{ACKNOWLEDGEMENT}

The author thanks the Department of Biotechnology, New Delhi for financial support to conduct this study.

\section{REFERENCES}

Akgun, I. and Tosum M. (2004). Agricultural and cytological characteristics of M1 rye Secale montanum Guss. as effected by the application of different doses of gamma rays. Pakistan Journal of Biological Science. 7(5): 827833.

Chaturvedi, H. C., Singh, S. K., Sharma, A. K. and Agnihotri, S. (2001). Citrus tissue culture employing vegetative explants. Indian Journal of Experimental Biology. 39:1080-1095.

Datta, S. K., Misra, P. and Mandal, A. K. A. (2005). In vitro mutagenesis- a quick method for establishment of solid mutant in chrysanthemum.Current Science 88 (1): 155158.

Davis,F. S. and Albrigo. I. G. (1994). Citrus. CAB International, Wallingford, UK: 254.

Dhatt, A. S., Rattanpal, H. S., Khokhar, R. and Singh, S. N. (2000). Standardising $L_{50}$ dose of gamma rays and EMS in Kinnow seeds. Journal of Research Punjab Agricultural University. 37 (3-4): 194-197.

Gad, A. A. and El-Sawah.,M. H. (1985). Comparative study on the relative mutagenic effectiveness and efficiency of gamma rays and some alkylating agents in peas. Egyptian Journal of Genetics and Cytology. 14(2):213-220.

Garcia-Agustin, P. and Primo-Millo, E. (1995). Selection of a NaCl tolerant citrus plant. Plant Cell Report. 14: 314318.

Gilchrist, E. J. and Haughn, G.W. (2005). Tilling without a plough: a new method with applications for reverse genetics. Current Opinion in Plant Biology. 8: 211-215.

Gulsen, O., Uzun, A., Pala, H., Canihos, E. and Kafa, G. (2007). Development of seedless and mal secco tolerant mutant lemons through budwood irradiation. Scientia Horticulturae. 112 (2): 184-190.

Gupta, S. C. and Sharma, K. D. (1994). Mutation induced variability in rice. Madras Agricultural Journal.81(1): 50-52.

Hearn, C. J. (1984). Development of seedless orange and grapefruit cultivars through seed irradiation. Journal of the American Society for Horticultural Science. 109 (2): 270-273.

Hensz, R. A. (1971). Star Ruby, a new red fleshed grapefruit with distinct tree characters. Journal of the Rio Grande Valley Horticultural Society. 14: 21-25.

Jain, S. M. (2005). In vitro mutagenesis for improving date palm (Phoenix dactylifera L.) . Emirates Journal of Food and Agriculture. 24:400-407.

Jander, G., Baerson, S. R., Hudak, J. A., Gonzalez, K. A., Gruys, K. J., Last, R. L.(2003). Ethyl methane sulfonate saturation mutagenesis in Arabidopsis to determine frequency of herbicide resistance. Plant Physiology.131: 139-146.

Jawaharlal, M.,Sambandamorthy, S. and Irulappan, I. (1992). Effect of gamma ray and EMS on seed germination and seedling growth in acidlime Citrus aurantifolia Swingle. South Indian Horticulture. 369: 332-336.

Kaur, S. and Rattanpal, H.S.(2010). Effect of mutagens on in vitro seed germination and growth of rough lemon (Citrus jambhiri Lush.) seedlings. Indian Journal of Agricultural Sciences. 80(9):773-776.

Kawamura, Y., Sadao Uchiyama, Yakio Saito. (1989). A half embryo test for identification of gamma irradiated grapefruit. Journal of Food Science. 54 (2): 379-382.

Kayim, M. and Koe, N. K. (2006). The effects of some carbohydrates on growth and somatic embryogenesis in citrus callus culture. Scientia Horticulture. 109: 29-34.

Kerkadze, I. G. (1985). Induced mutations in subtropical crops. V. Biological and genetic effect of treating citrus with gamma-radiation. Subtrop Kult. 4: 104-110.

Khan, M. R., Qureshi. A. S., Syed, A. H. and Ibrahim. M. (2005). Genetic variability induced by gamma irradiation and its modulation with gibbrellic acid in M2 generation of Chickpea (Cicer arietinum L.). Pakistan Journal of Botany.37(2):285-292.

Khokhar, R. (1998). 'Studies on mutagen induced variability in Kinnow mandarin.' MSc. thesis. Punjab Agricultural University, Ludhiana.

Kim, Y., Schumaker, K. S. and Zhu, J. K.(2006). EMS mutagenesis of Arabidopsis. Methods in Molecular Biology .323: 101-103.

Kumar, A. and Mishra, M. N. (2004). Effect of gamma rays, EMS and NMU on germination, seedling vigour, pollen viability and plant survival in M1 and M2 generation of Okra ( Abelmoschus esculentus L. Moench.). Advances in Plant Science. 17(1): 295-297.

Latado, R. R., TulmannNeto, A., Ando A Iemma, A. F., Pompeu Junior, J., Figueiredo, J. O., Pio, R. M., Machado, M. A., Namekata, T., Ceravolo, L. and Rossi, A. C. (2001). Sweet orange 'Pera' mutants with low number of seeds obtained through mutation induction. RevistaBrasileira de Fruticultura. 23 (2): 339-344.

Latado, R. R., Tulmann Neto, A. T., Pompeu Junior, J., Figueiredo, J. O., Pio, R. M., Machado, M. A., Namekata, T., Ceravolo, L., Montes, S. M. N. M. and Rossi, A. C. (2006). Seedless and citrus canker tolerant mutant clones in sweet orange induced by gamma rays. Plant Mutation Reports. 1 (2): 21-22.

Legave, J. M., Tissue-Agostini, D. and Jacquemond, C. (1989). Physiological effects induced by acute gammairradiation of clementine, Citrus reticulata Blanco budsticks. Fruits Paris. 446: 329-333.

Ling, A. P. K., Chia, J. Y., Hussein, S. and Harun, A. R. (2008). Physiological responses of Citrus sinensis to gamma irradiation. World Applied Sciences Journal. 5 (1):12-19.

MacKenzie, J. L., Saade, F. E., Le, Q. H., Bureau, T. E., Schoen, D. J .(2005). Genomic mutation in lines of Arabidopsis thaliana exposed to ultraviolet-B radiation. Genetics. 171: 715-723.

Majeed, A., Khan, A. U. R., Ahmad, H. and Muhammad, Z. (2010). Gamma irradiation effects on some growth parameters of Lepidium sativum L. ARPN Journal of Agricultural and Biological Science. 5 (1): 39-42.

Mallick, E. H., Hajar, N. G. and Bairagi, P. (1978). Physiological and biochemical mutagens, a priority to induced 
mutation. Indian Journal of Experimental Biology. 13: 187-188.

Murashige, T. and Skoog, F. (1962). A revised medium for rapid growth and bioassay with tobacco tissue culture. Plant Physiology. 15: 473-497.

Raghami, M. and Ghazvini, R. F. (2005). Effects of gamma irradiation on embryogenic callus and embryogenesis from habituated calluses of lime (Citrus aurantifolia var. Mexican lime). Iranian Journal of Agricultural Sciences. 36 (2): 425-434.

Ribeiro, R. V. and Machado, E. C. (2007). Some aspects of citrus ecophysiology in sub-tropical climates: revisiting photosynthesis under natural conditions. Brazilian Journal of Plant Physiology. 19:393-411.

Saini, H.K. and Gill, M. I. S.(2009). Induction of mutation in Rough lemon (Citrus jambhiri Lush.) using gamma rays. Journal of Horticultural Sciences. 4(1):41-44.

Sharma, L. K., Kaushal, M., Gill, M. I. S. and Bali, S. K. (2013). Germination and survival of Citrus jambhiri seeds and epicotyls after treating with different mutagens under in vitro conditions. Middle East Journal of Scientific Research. 16(2):250-255.

Sharma, G. and Sharma, R. L. (1996). Radio-sensitivity determination of $\mathrm{LD}_{50}$ in different apple cultivars. Haryana Journal of Horticultural Sciences. 251: 1-4.

Singh, S., Bansal, M. L., Singh, T. P. and Kumar, P.(1998). Statistical Methods for Research Workers. Kalyani Publishers, New Delhi.

Singh, R. R. and Singh, J. (1989). Genotypic variability relationship with EMS in a Trigonella species complex. Indian Journal of Horticulture. 461: 111-114.

Sparrow, A. H. and Gunckel, J. E. (1956). The effect of plants on chronic exposure to gamma radiation from radio Cobalt. Proceedings of International Conference on Peaceful Uses of Atomic Energy. Pp. 52-59.

Swaminathan, M. S. (1965). A comparison of mutation induction in diploids and polyploids. In: The use of mutations in plant breeding. Radiation Botany (Supplement). 5: 619-641.

Swaminathan, M. S., Chopra, V. L. and Bhaskaran, S. (1962). Chromosome aberrations and the frequency and spectrum of mutations induced by ethyl methane sulfonate in barley and wheat. Indian Journal of Genetics. 22: 192-207.

Thapa, C. B.(2004). Effect of acute exposure of gamma rays on seed germination and seedling growth of Pinus kesiya Gord and Pinus wallichiana A.B. Jacks. Our nature. $2: 13-17$.

Toker, C., Uzen, B., Canci, H. and Ceylan, F. O. (2005).Effect of gamma irradiation on the shoot length of Cicer seeds. Radiation Physics and Chemistry.73:365 $-367$.

Wan, S. Y., Deng, Z., Deng, X. X., Ye, X. and Zhang, W. C. (1991). Advances made in in vitro mutation breeding in citrus. Proceedings of International Symposium on the Contribution of Plant Mutation Breeding to Crop Improvement, Vienna, Austria. 1: 263-269.

Veleminsky, J. ,Gichner, T. and Pokorny,V.(1975). Caffeine enhancement of alkalyting agent-induced injury in barley: its connection to DNA single-strand and their repair. Mutation Research. 28(1):79-86.

Waqar, A., Wasin, A., Farooqi and Sattar, S. Jr. (1992). Effect of gamma-irradiation on the morphology of Kinnow seedlings. Proceeding of the First International Seminar on Citriculture in Pakistan 2-5 December, 1992.

Whittwer, S H. (1971). Radiation induced mutations in crop plants. Outlook Agriculture. 6: 205-217. 